\title{
Étude des équilibres des phases du carbonate de calcium : influence des compagnons de cristallisation
}

\author{
A.S. Manzola et M. Ben Amor \\ Laboratoire des Procédés Chimiques, Institut National de Recherche Scientifique et Technique, \\ BP. 95, 2050 Hammam-Lif, Tunisia
}

\begin{abstract}
Résumé . Les eaux naturelles, en particulier dans le sud tunisien très chargées en sels, ont une dureté très élevée. Elles sont le siège d'une précipitation importante de $\mathrm{CaCO}_{3}$ dans les conduites d'adduction. La phase précipitée est l'aragonite dans les eaux refroidies à $30^{\circ} \mathrm{C}$. La littérature précise que cette phase doit précipiter préférentiellement à des températures $\geq 50^{\circ} \mathrm{C}$. C'est la raison pour laquelle nous avons repris l'étude de l'influence des compagnons de cristallisation : $\mathrm{Na}^{+}, \mathrm{Cl}^{-}, \mathrm{Mg}^{2+}$, $\mathrm{Ca}^{2+}$ et $\mathrm{SO}_{4}{ }^{2-}$ à $30{ }^{\circ} \mathrm{C}$. Le $\mathrm{Mg}^{2+}$ favorise l'apparition de l'aragonite tandis que le $\mathrm{SO}_{4}{ }^{2-}$ et le $\mathrm{Ca}^{2+}$ favorisent la formation de la calcite. Les ions $\mathrm{Na}^{+}$et $\mathrm{Cl}^{-}$n'ont pas d'effet sur la nature de la phase précipitée.
\end{abstract}

\section{INTRODUCTION}

La formation de dépôts de tartre, constitué essentiellement de carbonate de calcium, est un problème majeur dans les installations industrielles ou domestiques, alimentées en eaux naturelles ou de distribution. Pour prévenir ou lutter contre ce problème plusieurs études ont été menées sur le système carbonique et calcocarbonique en fonction de la température : $\mathrm{CaCO}_{3}, \mathrm{CO}_{2}, \mathrm{H}_{2} \mathrm{O}$. L'étude de ce système triphasique devient de plus en plus complexe si on tient compte, d'une part de la composition très variée des eaux naturelles et d'autre part des phases cristallographiques du carbonate de calcium.

Nous illustrons ce phénomène par le cas de l'entartrage des eaux géothermales du sud de la Tunisie. En effet, compte tenu de la rareté de l'eau dans le sud et pour lutter contre la désertification, le Gouvernement a mis en exploitation une nappe d'eaux fossiles de profondeur variant entre 2000 et 3000 mètres. Le débit est compris entre 80 et $1001 . \mathrm{s}^{-1}$, soient en moyenne $7800 \mathrm{~m}^{3} \mathrm{j}^{-1}$. La température de l'eau à la sortie varie entre 50 et $73^{\circ} \mathrm{C}$. Les eaux géothermales sont très chargées en calcium et en magnésium de concentrations respectives 400 et $100 \mathrm{mg} \cdot \mathrm{I}^{-1}$ et ont une salinité supérieure à $2,5 \mathrm{~g} .1^{-1}$ [1]. Ces eaux, refroidies dans des systèmes réfrigérants atmosphériques, sont utilisées principalement pour l'irrigation. Il se produit un phénomène d'entartrage qui bouche les circuits de distribution des eaux et les systèmes réfrigérants. Nous présentons, ci-joint, une coupe d'une conduite bouchée, le précipité est de l'aragonite malgré que les eaux soient refroidies à $30^{\circ} \mathrm{C}$.

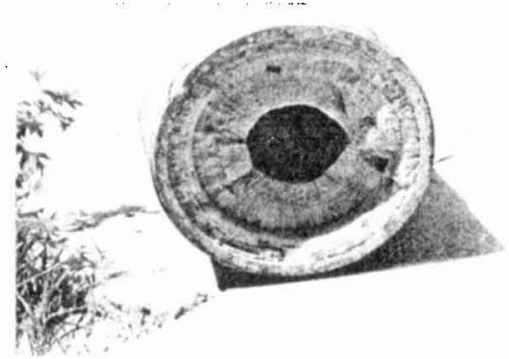

Figure 1 : Conduite d'eau géothermale entartrée. 
Compte tenu du fait que les ions les ions $\mathrm{Mg}^{2+}, \mathrm{SO}_{4}{ }^{2-}, \mathrm{Ca}^{2+}, \mathrm{Na}^{+}$et $\mathrm{Cl}^{-}$sont présents dans les eaux géothermales, nous nous sommes intéressés particulièrement à l'étude de l'effet de ces ions sur les phases polymorphiques de $\mathrm{CaCO}_{3}$ formées. Les ions $\mathrm{Mg}^{2+}, \mathrm{SO}_{4}{ }^{2-}, \mathrm{Ca}^{2+}, \mathrm{Na}^{+}, \mathrm{Cl}^{-}$ affectent la cristallisation du carbonate de calcium $[2,3,10]$.

\section{2- LE CARBONATE DE CALCIUM}

Le tartre est essentiellement constitué de carbonate de calcium, mais il renferme également, selon l'origine de l'eau ou de la nature des canalisations et leur état de corrosion, d'autres composés peu solubles, par exemple $\mathrm{CaSO}_{4}, \mathrm{Fe}(\mathrm{OH})_{3}, \mathrm{SiO}_{2}, \mathrm{MgCO}_{3}, \mathrm{Al}(\mathrm{OH})_{3}$.

La présentation de quelques propriétés physico-chimiques du carbonate de calcium contribue à la compréhension du phénomène d'entartrage.

Il existe trois variétés cristallographiques de carbonate de calcium :

- La calcite: cristallisant dans le système rhomboédrique, c'est la variété thermodynamiquement la plus stable à la température ambiante. L'unité du réseau de la calcite est un rhomboèdre aigu, contenant deux molécules de $\mathrm{CaCO}_{3}$ par maille..

- L'aragonite: cristallisant dans le système orthorhombique, c'est une variété métastable à la température ambiante ; mais devenant la variété thermodynamiquement la plus stable audessus de $60^{\circ} \mathrm{C}$. Elle se présente sous forme d'aiguilles fines. L'unité du réseau de l'aragonite est un parallélépipède contenant quatre molécules de $\mathrm{CaCO}_{3}$ par maille.

- La vatérite: cristallisant dans le système hexagonal est beaucoup plus instable que les deux formes précédentes et qui évolue très facilement et très rapidement vers l'aragonite. La vatérite se présente soit sous forme d'aiguilles trapues, avec des articulations, soit sous forme de 'fleurs' ou 'lentilles'. Elle est obtenue en présence d'un excès de calcium $[11,12,14]$

Il existe aussi sous deux formes hydratées :

Le carbonate de calcium monohydraté : cristallisant dans le système hexagonal est obtenu en présence de $\mathrm{Mg}^{2+}$ ou d'autres ions d'eau de mer ou des matières organiques [4].

Le carbonate de calcium hexahydraté : cristallise dans le système monoclinique aussi bien en milieu naturel qu'au laboratoire à une température proche de $0^{\circ} \mathrm{C}$ et se transforme en phase anhydre à la température ambiante[5].

\section{ETUDE EXPERIMENTALE}

Dans cette étude, nous avons utilisé le montage de dégazage contrôlé mis au point par H. ROQUES [6], puis amélioré par la suite par [7]. Cette technique vise à dissocier les différentes étapes cinétiques pour isoler celle qui est la plus limitante. L'ensemble des manipulations s'effectue sous atmosphère gazeuse de composition connue de façon à contrôler parfaitement les variations de concentration en $\mathrm{CO}_{2}$ dissous qui constituent le principe moteur de l'évolution du système selon la réaction fondamentale :

$$
\mathrm{Ca}^{2+}+2 \mathrm{HCO}_{3}^{-} \rightleftarrows \mathrm{CaCO}_{3}(\mathrm{~s})+\mathrm{CO}_{2}+\mathrm{H}_{2} \mathrm{O}
$$

Cette réaction résulte des deux réactions, une première réaction d'élévation du $\mathrm{pH}$ :

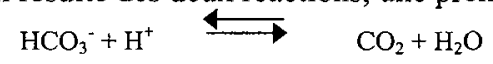

En effet le dégazage du $\mathrm{CO}_{2}$ déplace l'équilibre dans le sens de la consommation des $\mathrm{H}^{+}$, donc vers une élévation du $\mathrm{pH}$. La précipitation de $\mathrm{CaCO}_{3}$ qui se traduit par une chute du $\mathrm{pH}$ selon la deuxième réaction :

$$
\mathrm{Ca}^{2+}+\mathrm{HCO}_{3}^{-} \longrightarrow \mathrm{CaCO}_{3}(\mathrm{~s})+\mathrm{H}^{+}
$$

Les solutions calcocarboniques pures ont été préparées par dissolution du $\mathrm{CaCO}_{3}$ dans l'eau déminéralisée sous barbotage du dioxyde de carbone pendant une dizaine d'heures. Les essais ont été réalisés dans une cellule en PVC à $30^{\circ} \mathrm{C}$. Le gaz de travail est de l'air 
atmosphérique de façon à se rapprocher au maximum des conditions réelles. La solution calcocarbonique étudiée avait une concentration initiale de $300 \mathrm{mg} / \mathrm{l}$ de $\mathrm{CaCO}_{3}$. Nous mesurons à la fois le $\mathrm{pH}$ et la concentration en hydrogénocarbonate. La manipulation est arrêtée 3 minutes après le début la chute du $\mathrm{pH}$; le solide est filtré à travers une membrane millipore de $0,45 \mu \mathrm{m}$ puis séché à $333 \mathrm{~K}$ à l'étuve pendant 10 minutes.

La diffraction des Rayons X, la microscopie électronique à balayage (MEB) et la spectroscopie infrarouge permettent de bien distinguer les phases précipitées $[8,9,10]$.

\section{DISCUSSIONS ET INTERPRETATIONS DES RESULTATS}

\subsection{Résultats expérimentaux}

Nous avons étudié l'influence des ions $\mathrm{Na}^{+}, \mathrm{Mg}^{2+}, \mathrm{Ca}^{2+}, \mathrm{SO}_{4}{ }^{2-}$ et $\mathrm{Cl}^{-}$sur la croissance cristalline des différentes phases de $\mathrm{CaCO}_{3}$.

\section{$3.1 .1 \mathrm{NaCl}$}

$\mathrm{Vu}$ la forte augmentation de la force ionique $(\mu)$ qu'entraîne l'ajout de $\mathrm{NaCl}$, nous avons fait 4 essais comparatifs à $0 ; 0,01 ; 0,02$ et 0,03 mol. $^{-1}$ de NaCl. $(\mu=0,009 ; 0,019 ; 0,029 ; 0,039)$. Les diffractogrammes des Rayons $X$, les spectres infrarouge et la photo MEB des solides précipités sont représentés sur les figures $2 \mathrm{a}, 2 \mathrm{~b}$ et 6 .

Les diffractogrammes des Rayons X (figures 2a. et 2b.) sont quasi-identiques. Les photos MEB des solides formés, qui confirment ces résultats, montrent des lentilles ou de "rose de sable", des rhomboèdres et des amas d'aiguilles (photo 1, figure 6). Les spectres infrarouge sont parfaitement identiques en absence et en présence de $\mathrm{NaCl}$. Ils montrent la présence des pics d'absorption à $1082,872,852,745,710$ et $699 \mathrm{~cm}^{-1}$.
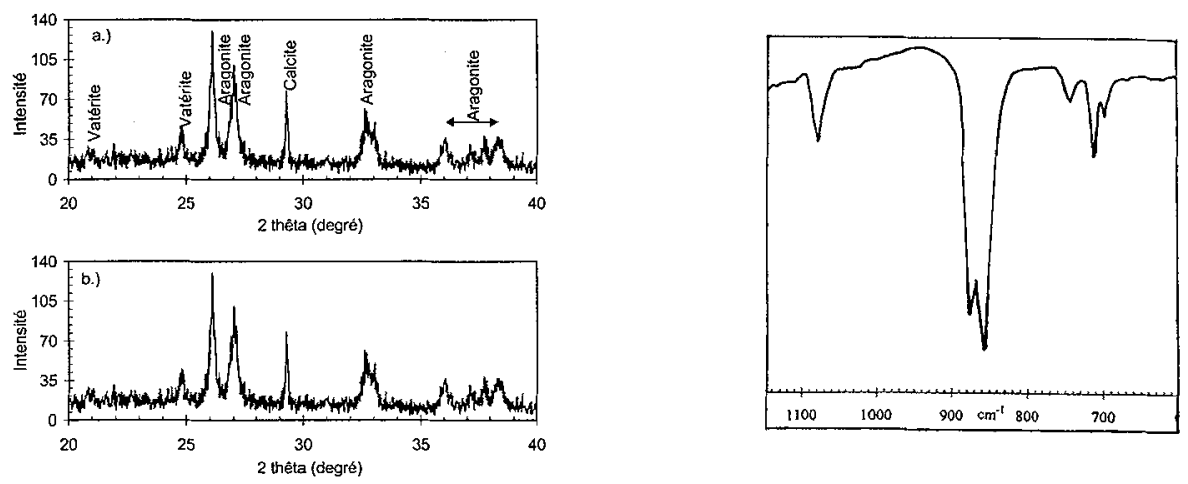

a) $\mathrm{NaCl}=0,00 \mathrm{M}$; b.) $\mathrm{NaCl}=0,03 \mathrm{M}$

Figure 2 : Diffractogrammes des Rayons $\mathrm{X}$ et spectres infrarouges des produits précipités :

\subsection{2 $\mathrm{Mg}^{2+}$}

Le magnésium a été introduit sous forme de $\mathrm{MgCl}_{2}$ puisque l'ion $\mathrm{Cl}^{-}$n'a pas d'effet comme il a été montré dans le cas précédent. Les concentrations de $\mathrm{Mg}^{2+}$ étudiées sont : $0 ; 24,3 ; 60$; 120 et $242 \mathrm{mg} \mathrm{.l^{-1 }}$. La figure 3 illustre les résultats obtenus.

Sur le diffractogramme des Rayons X (figure $3 \mathrm{c}$ ) les pics correspondant à la calcite diminuent lorsque la concentration en magnésium augmente. De même, le spectre infrarouge (figure 3) montre que seuls les pics d'absorption à 1082, 852, 710 et $699 \mathrm{~cm}^{-1}$ correspondant à l'aragonite subsistent. Le même résultat se retrouve sur les photos MEB (photo 2 et 3 , figure 6). Nous observons une forte présence d'amas d'aiguilles. On confirme que le magnésium favorise la formation de l'aragonite à la température de $30^{\circ} \mathrm{C}$. 

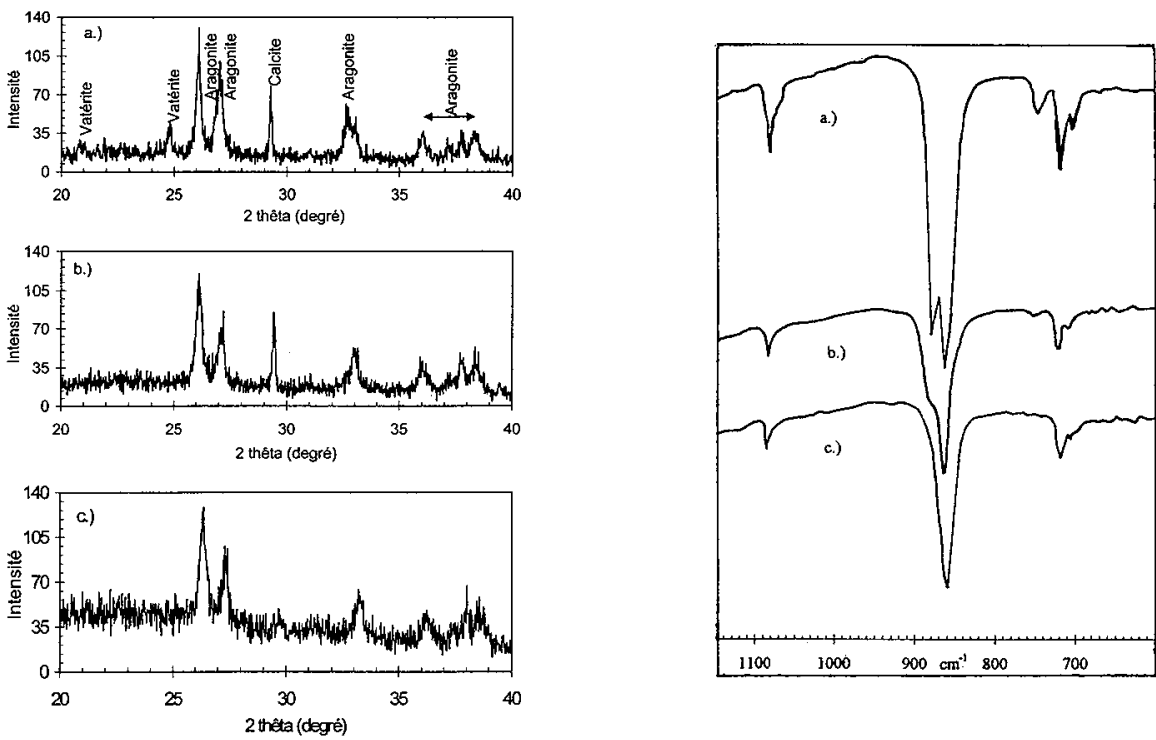

a.) $\mathrm{Mg}=0$; b.) $\mathrm{Mg}=120 \mathrm{mg} / \mathrm{l} ; \mathrm{Mg}=240 \mathrm{mg} / \mathrm{l}$.

Figure 3 : Diffractogrammes des Rayons $\mathrm{X}$ et spectres infrarouges des précipités en présence du magnésiur.

\subsection{3 $\mathrm{SO}_{4}{ }^{2-}$}

L'ion $\mathrm{SO}_{4}{ }^{2-}$ a été introduit sous forme de $\mathrm{Na}_{2} \mathrm{SO}_{4}$ puisqu'on a vu que l'ion $\mathrm{Na}^{+} \mathrm{n}$ 'a $\mathrm{p}$ d'effet.
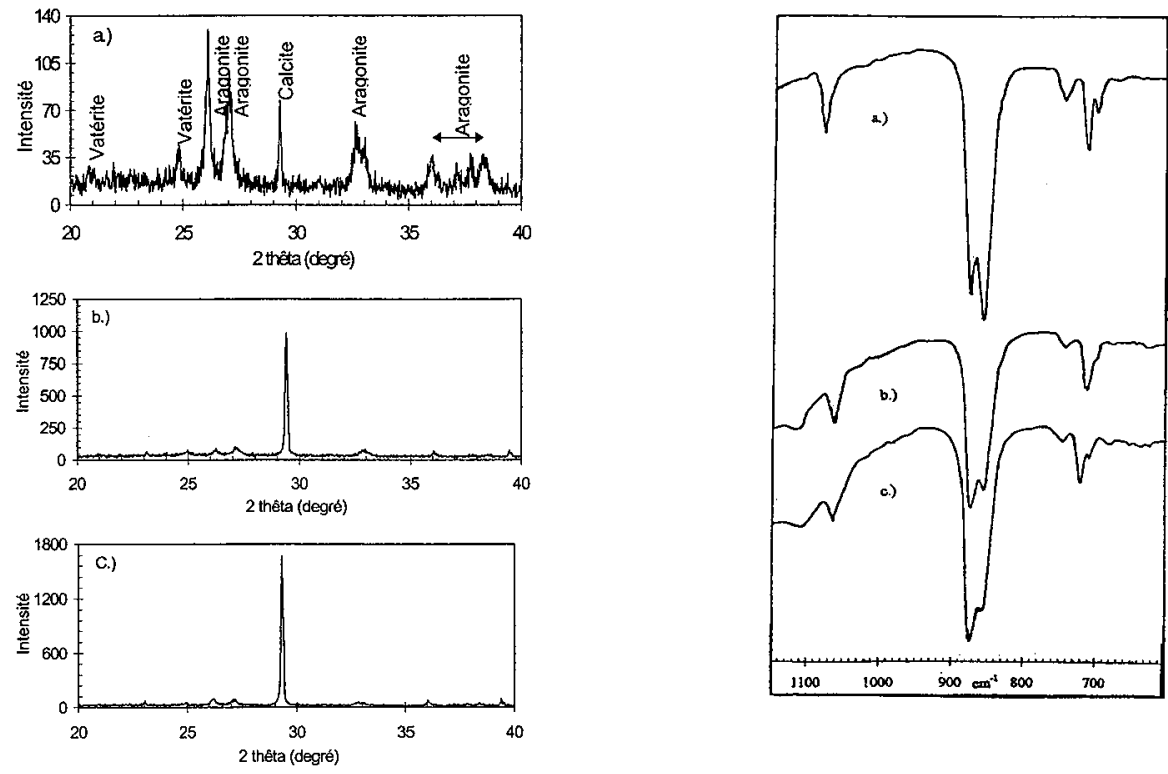

a.) $\mathrm{SO}_{4}{ }^{2-}=0,00 \mathrm{mg} / \mathrm{l} ;$ b.) $\mathrm{SO}_{4}{ }^{2-}=300 \mathrm{mg} / 1 ; \mathrm{SO}_{4}{ }^{2-}=600 \mathrm{mg} / 1$.

Figure 3 : Diffractogrammes des Rayons $\mathrm{X}$ et spectres infrarouges des produits précipités. 
Les concentrations de $\mathrm{SO}_{4}{ }^{2-}$ étudiées sont $0,300,600,900$ et $1200 \mathrm{mg} / 1$. Les figures 4 et 6 illustrent les résultats obtenus.

Sur la figure 4 les diffractogrammes des Rayons X ( $b$ et $\mathrm{c}$ ) montrent que le précipité est bien cristallisé par rapport aux précédents, avec une forte prédominance du pic caractéristique de la calcite. De même, sur le spectre infrarouge correspondant, le pic caractéristique de l'aragonite diminue et celui de la calcite augmente. La photo MEB correspondante (photo 4, figure 6) montre pratiquement que des rhomboèdres.

\subsection{4 $\mathrm{Ca}^{2+}$}

Le $\mathrm{Ca}^{2+}$ est introduit sous forme de $\mathrm{CaCl}_{2}$, puisque l'ion $\mathrm{Cl}^{-} \mathrm{n}$ 'est pas actif comme on l'a déjà remarqué. Les concentrations étudiées sont : 0, 40,100,200, 400 et $600 \mathrm{mg} / 1$. Les résultats sont représentés sur la figure 5.
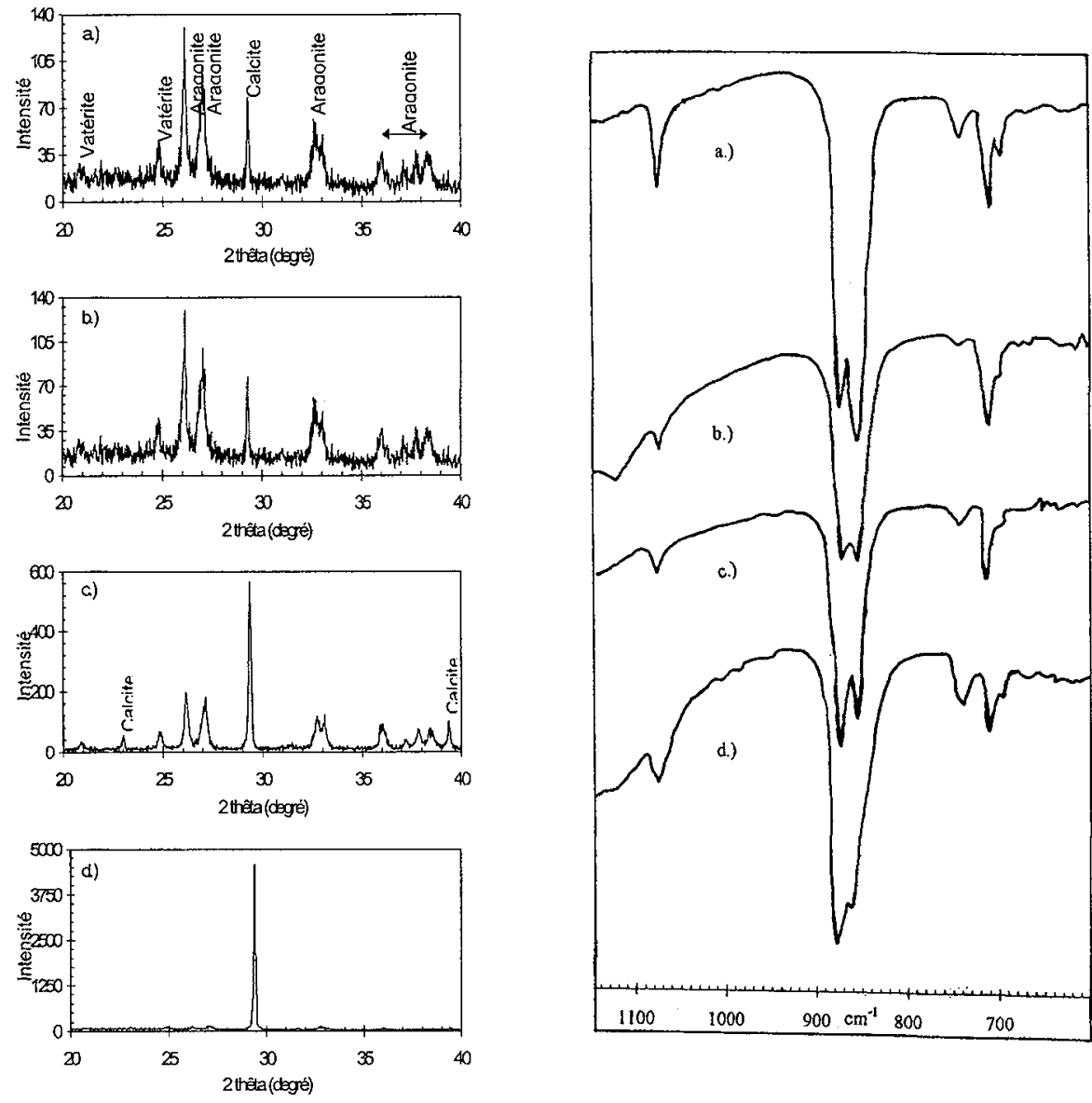

a.) Ca perm. $=0 \mathrm{mg} / \mathrm{l} ;$ b.) Ca perm. $=100 \mathrm{mg} / \mathrm{l}$; c.) Ca perm. $=400 \mathrm{mg} / \mathrm{l} ;$ d.) Ca perm. $=600 \mathrm{mg} / \mathrm{l}$. Figure 5 : Diffractogrammes des Rayons $X$ et spectres infrarouges des produits précipités :

Les diffractogrammes de Rayons X ( b. et c.) montrent l'amélioration de la cristallinité des précipités, ce qui se reflète par l'augmentation des intensités relatives de 140 à 600 puis à 
5000. On note aussi, la prédominance de la calcite. Le spectre infrarouge ( b.) montre une coexistence à part égale entre la calcite et l'aragonite. Sur le spectre infrarouge (c) la phase aragonite devient de plus en plus petite et tend à disparaître, tandis que la calcite apparaît pratiquement seule. La photo MEB correspondante (photo 5, figure 6) montre également la prédominance des rhomboèdres avec un peu plus de lentilles et de "rose de sable", et quelques amas d'aiguilles résiduels.

La photo MEB correspondante (photo 6, figure 6) montre une quasi-prédominance des rhomboèdres et confirme le résultat de prédominance de la calcite avec l'augmentation de la concentration du calcium permanent dans l'eau.

\subsection{Interprétations et discussions}

Concernant l'influence du $\mathrm{NaCl}$, les diffractogrammes de Rayons $\mathrm{X}$ sont pratiquement identiques en présence ou non de chlorure de sodium (figures $2 \mathrm{a}$ et $2 \mathrm{~b}$ ). On trouve les 3 variétés cristallographiques vatérite, calcite et l'aragonite qui prédomine. Les observations au MEB (photo 1, figure 6) des solides formés confirment ces résultats.

Les spectres infrarouge des solides précipités en absence ou en présence de tout ion étranger au système calcocarbonique montrent à $745 \mathrm{~cm}^{-1}$ la présence de la vatérite, à 872 $\mathrm{cm}^{-1}$ la présence de la calcite à $1082,852,710$ et $699 \mathrm{~cm}^{-1}$ la présence de l'aragonite. Le spectre infrarouge et la photo MEB présentés sont identiques à ceux obtenus en présence de $\mathrm{NaCl}$. Donc nous pouvons affirmer que les ions $\mathrm{Na}^{+}$et $\mathrm{Cl}^{-} \mathrm{n}^{\text {'ont }}$ pas d'effet sur la variété cristallographique formée comme 1'ont montré Roques et El Fil [11, 12] et Zidoune [13] avec la technique de la chronoélectrogravimétrie.

Pour le $\mathrm{Mg}^{2+}$, sur la figure 3, le diffractogramme des Rayons X ( b ) fait apparaître la présence de l'aragonite prédominante et de la calcite. Il n' y a aucun pic de la vatérite. Sur la photo MEB correspondante (photo 2, figure 6) nous observons la présence de l'aragonite prédominante, de la calcite résiduelle, mais aussi de la vatérite en cours de transformation en aragonite.

Le spectre infrarouge (figure 3) correspondant montre la présence des pics des trois variétés existantes. Sur ce spectre le pic d'absorption de la vatérite existe, ce qui est en accord avec les observations des photos MEB qui montrent la présence de la vatérite en cours de transformation. Donc à partir de $120 \mathrm{mg} / \mathrm{l} \mathrm{de} \mathrm{Mg}^{2+}$, la vatérite se transforme directement en aragonite, ce qui donne de la vatérite mal cristallisée, donc non détectable aux Rayons X. Le pic $872 \mathrm{~cm}^{-1}$ qui correspond à la calcite diminue d'intensité et tend à se confondre avec le pic $852 \mathrm{~cm}^{-1}$ de l'aragonite. En (c. ), seuls les pics d'absorption de l'aragonite subsistent et sur la photo MEB correspondante (photo 3, figure 6) seuls les amas d'aiguilles d'aragonite sont présents.

Ces résultats sont en parfait accord avec ceux obtenus par Roques à $30^{\circ} \mathrm{C}$ [11] pour des concentrations comprises entre 0 et $1100 \mathrm{mg} / 1$ et pour une solution calcocarbonique de $200 \mathrm{mg} / \mathrm{l}$, il observe l'apparition des cristaux de calcite magnésienne, devenant de plus en plus magnésienne avec l'augmentation de la concentration en $\mathrm{Mg}^{2+}$. Pour des concentrations supérieures, il observe que l'ion $\mathrm{Mg}^{2+}$ inhibe la formation des cristaux de la calcite et favorise la formation d'aragonite. Il a observé aussi une augmentation très nette de la vitesse des transformations des lentilles de vatérite en aragonite.

Donc on conclut à l'aide de cette étude que la présence du magnésium à fortes concentrations favorise la formation de l'aragonite.

Concernant l'ion sulfate les données de la littérature sont assez contradictoires. Roques [11] observe que l'ion $\mathrm{SO}_{4}{ }^{2-}$ favorise légèrement la formation de la calcite et inhibe la transformation des lentilles de vatérite. Sur la figure 4 le diffractogramme des Rayons X ( b.) 
montre une quasi-prédominance de la calcite avec toutefois une coexistence de l'aragonite et de la vatérite. Le diffractogramme des Rayons X ( c.) montre une forte prédominance de la calcite. Sur le spectre infrarouge correspondant, le pic d'absorption de l'aragonite diminue d'intensité, par contre celui de la calcite a augmenté.. La photo MEB correspondante (photo 4, figure 6) montre pratiquement que de la calcite. Ce résultat a été observé par El Fil [12] et Zidoune [13] par la technique de la chronoélectrogravimétrie.

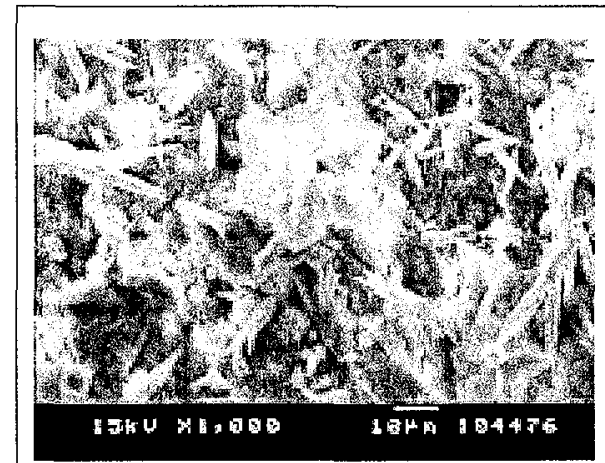

Photo 1

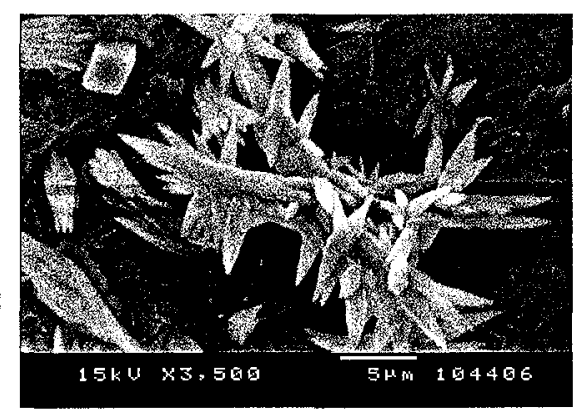

Photo 2

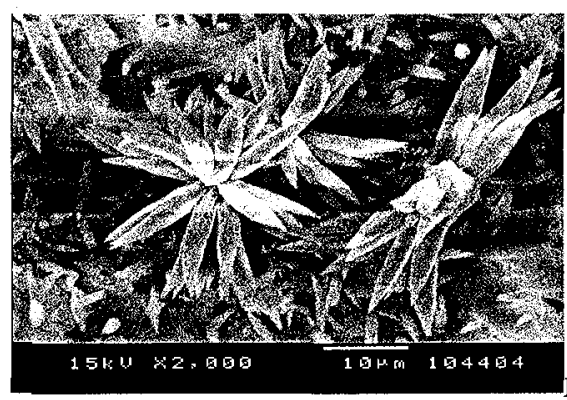

photo 3

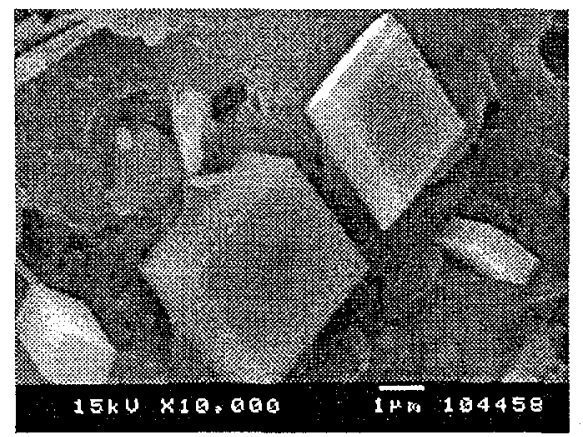

Photo 4

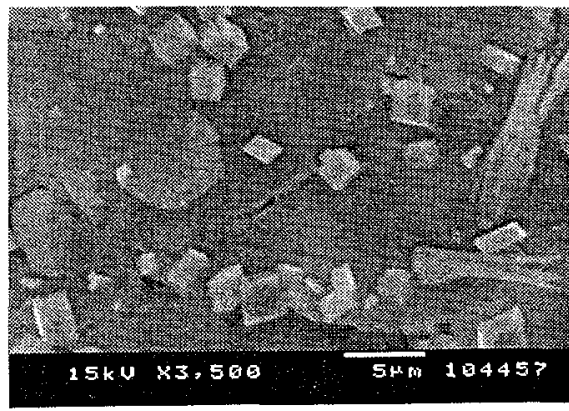

Photo 5

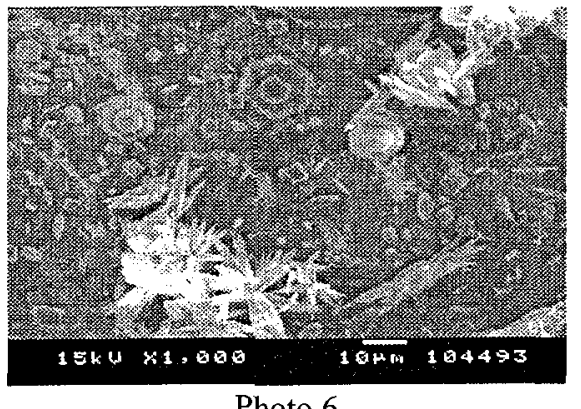

Photo 6

Figure $6:$ Photos MEB

Les ions $\mathrm{Ca}^{2+}$ en excès favorisent la formation de la vatérite à des températures inférieures à $40^{\circ} \mathrm{C}$ [14]. Sur la figure 5 le diffractogramme des Rayons X (b. et c. ) montre une prédominance de la calcite, une faible quantité de la vatérite et de l'aragonite résiduelle. A une température de $50^{\circ} \mathrm{C}$ pour des fortes concentrations en $\mathrm{Ca}^{2+}$, El Fil [12] observe que la calcite disparaît et ne subsistent plus que de la vatérite et de l'aragonite. 
La photo MEB correspondante (photo 5, figure 6) montre également de la calcite prédominante avec effectivement de la vatérite, et un peu d'aragonite. Le spectre infrarouge (b. ) montre une coexistence à part égale entre la calcite et l'aragonite, alors que le diffractogramme des rayons X correspondant montre une prédominance de l'aragonite. Le pic d'absorption de la calcite devient plus important que celui de l'aragonite en ( c. ). Le diffractogramme des Rayons X ( d. ) qui correspond à la forte concentration en $\mathrm{Ca}^{2+}$ montre une quasi-prédominance de la calcite. La photo MEB correspondante (photo 6, figure 5) confirme ce résultat, mais avec plus de vatérite. Sur le spectre infrarouge correspondant le pic de l'aragonite devient encore plus petit et tend à se confondre à celui de la calcite. Le pic 745 $\mathrm{cm}^{-1}$ a augmenté d'amplitude, ce qui est en accord avec la photo MEB.

\section{CONCLUSIONS}

Cette étude montre l'importance des compagnons de cristallisation sur la nature de la phase du carbonate de calcium précipité. En effet, pour bien prévenir le phénomène de précipitation, nous devons tenir compte de la composition de l'eau à étudier surtout par les moyens physiques : magnétique et électromagnétique, qui consistent à précipiter préférentiellement des phases moins incrustantes. On cite l'exemple d'un traitement électromagnétique qui consiste à précipiter la phase aragonite dans des eaux riches en sulfate pourrait être inefficace.

\section{Références}

[1] WALHA K. : "Procédés de lutte contre l'entartrage par les eaux géothermales du sud tunisien" Thèse de Doctorat. Université de Sfax, Mai 1999.

[2] AKIN, G. W., LAGERWERFF, J. V., Calcium carbonate equilibria in aqueous solutions open to the air. II. Enhanced solubility of $\mathrm{CaCO}_{3}$ in the presence of $\mathrm{Mg}^{2+}$ and $\mathrm{SO}_{4}{ }^{2-}$. Geochim. Cosmochim. Acta 29, 1965, 353-360.

[3] MUCCI, A., ZHONG, J. W., The solubility of calcite and aragonite in sulphate-free seawater and the seeded growth kinetics and composition of the precipitates at $25^{\circ} \mathrm{C}$. Chem. Geol. 74, 1983, 309-320.

[4] KRALJ D. and BRECEVIC, Colloids Surf. A, 96 (1995) 287-293.

[5] BISCHOFF J. L., FITZATRICK J. A. and ROSENBUER R. J., J. Geol.,101 (1993) 21-33.

[6] ROQUES H., Contribution à l'étude statique et cinétique des systèmes gaz carbonique eau - carbonate. Thèse de Doctorat, faculté des sciences, Toulouse, $N^{\circ} 228$. France, 1964.

[7] HORT C., MARTIN-DOMINGUEZ A., RABUT C. et ROQUES H., Contribution à l'étude des phénomènes d'entartrage, Tribune de l'eau, 48 (1995) 31-46.

[8] ANDERSEN F. A. and BRECEVIC Lj., Infrared spectra of amorphous and cristalline calcium carbonate. Acta Chemica Scandinavica, 45(1991) 1018-1024.

[9] Powder diffraction file, Inorganic Phases, 1986, Joint Commitee on Powder Diffraction Standards, Swarthmore, PA, USA.

[10] ROQUES H., Fondements théoriques du traitement chimique des eaux". Vol. I et II. Technique et documentation, Lavoisier. Paris 1990.

[11] ROQUES H., Influence des ions étrangers et de la matière organique sur la cristallisation des carbonates de calcium, Revue de l'Institut Français du Pétrole, 34 (1979) N¹.79003.

[12] ELFIL H., Contribution à l'étude des Eaux Géothermales du Sud tunisien, I.N.S.A de Toulouse, N507. Février 1999. France

[13] ZIDOUNE M., Contribution à la connaissance des mécanismes d'entartrage par diverses méthodes électrochimiques, Thèse de Doctorat de l'Université Paris VI, 1996.

[14] OGINO T., SUZUKI T. \& SAWADA K., The formation and transformation mechanism of calcium carbonate in water. Geochimica et Cosmochimica Acta Vol. 51, 1987, 2757-2767. 